\title{
CSF gradients for amino acid neurotransmitters
}

\author{
P M CRAWFORD, K G LLOYD, D W CHADWICK \\ From the Mersey Regional Medical and Surgical Neurology Unit, Walton Hospital, Liverpool, UK
}

SUMMARY Amino acid concentrations were measured in CSF samples obtained by lumbar puncture in 51 patients, cervical puncture in 16 patients, spinal drains in nine patients, ventricular taps in five patients and from below a spinal block in six patients. There was evidence of a rostrocaudal gradient for GABA and taurine and a reverse gradient for alanine and asparagine. Lumbar CSF glycine concentrations rose with increasing age whilst GABA concentrations fell. Women had significantly lower concentrations of asparagine and glutamine and elevated taurine compared to men.

The influence of biological factors and gradients must be taken into account before the interpretation of changes in CSF amino acid concentrations.

There is increasing awareness of the role that central neurotransmitters may play in neurological disease. However, the opportunities to study neurotransmitter function in vivo are strictly limited. Over recent years methods for the assay of neurotransmitters and their metabolites have become available and have been applied to human lumbar CSF.

There have been many publications concerning CSF amino acid concentrations in health and disease. ${ }^{1-7}$ There are wide variations in control concentrations and considerable inter-individual variation amongst patients. Some of the variation may be due to small numbers in some of the control groups. Only two papers have control groups of over 20 patients. ${ }^{12}$

For metabolites of dopamine (homovanillic acidHVA) and serotonin (5-hydroxyindole acetic acid5H1AA) it can be shown that there is a rostrocaudal

Address for reprint requests: Dr D W Chadwick, Consultant Neurologist, Walton Hospital, Rice Lane, Liverpool L9 1AE, UK

Received 28 April 1987 and in revised form 21 April 1988. Accepted 26 April 1988 gradient for concentrations and that the conditions under which samples are collected and the age of the patient may influence results. ${ }^{8}$ Recently there has been interest in the amino acid neurotransmitters and their roles in disease. However, interpretation of results for lumbar CSF is difficult because a number of factors remain to be established: (1) Are conditions under which specimens are collected critical? (2) What are the relative contributions of spinal cord and cerebrum to amino acid neurotransmitter concentrations in the lumbar sac? (3) Do biological variables (age and sex) influence concentrations?

We report free CSF GABA and other amino acid neurotransmitter concentrations in CSF obtained from cervical and lumbar punctures, spinal drains and ventricular taps in an attempt to answer some of these questions.

\section{Methods}

A Patient characteristics

These are described in table 1.

Table 1 Patient characteristics

\begin{tabular}{|c|c|c|c|c|c|}
\hline \multirow[b]{2}{*}{ Origin of $C S F$} & \multirow[b]{2}{*}{$n$} & \multicolumn{2}{|c|}{ Age } & \multicolumn{2}{|c|}{ Sex } \\
\hline & & $\begin{array}{l}\text { Mean } \\
(y r, S D)\end{array}$ & $\begin{array}{l}\text { Range } \\
(y r)\end{array}$ & Male & Female \\
\hline \multicolumn{6}{|l|}{ Lumbar } \\
\hline $\begin{array}{l}\text { Myelograms } \\
\text { Neurological controls } \\
\text { Spinal block } \\
\text { Cervical } \\
\text { Ventricular } \\
\text { Spinal drains }\end{array}$ & $\begin{array}{r}37 \\
14 \\
6 \\
16 \\
5 \\
9\end{array}$ & $\begin{array}{l}48,15 \\
50,19 \\
66,9 \\
49,16 \\
49,24 \\
43,15\end{array}$ & $\begin{array}{l}14,73 \\
15,74 \\
48,73 \\
19,71 \\
13,71 \\
22,64\end{array}$ & $\begin{array}{r}19 \\
9 \\
4 \\
12 \\
3 \\
6\end{array}$ & $\begin{array}{r}18 \\
5 \\
2 \\
4 \\
2 \\
3\end{array}$ \\
\hline
\end{tabular}


1 - Lumbar Myelography Thirty seven patients underwent lumbar myelography for suspected disc prolapse. Nineteen were male and 18 female. Two patients had cauda equina lipomas, one patient had a tethered spinal cord, the rest either had normal myelograms or disc prolapses not causing obstruction to the flow of CSF. These patients had not received any drugs in the previous 24 hours.

2 Cervical myelograms Sixteen patients had a cervical puncture for myelography. Twelve were male, four female. Patients either had a minor cervical disc prolapse or a normal myelogram. Patients for myelography were not fasted prior to collection of CSF and had 30 minutes bed rest prior to the procedure. They were given $1 \mathrm{mg}$ of clonazepam orally 1 hour before myelography. CSF was collected from either a lumbar puncture at $\mathrm{L} 4 / 5$ or a lateral cervical puncture at $\mathrm{C1} / 2$. The first $3 \mathrm{ml}$ of CSF was sent for routine biochemical analysis and the next $5 \mathrm{ml}$ were collected for neurotransmitter analysis.

3 Spinal block CSF was obtained from below a block by lumbar puncture in six patients whose myelogram showed a complete block to the flow of CSF. Two patients had a complete block in the cervical region from a prolapsed cervical disc, the other four patients had mid thoracic blocks. Three of these four patients had extradural deposits from a carcinoma or a sarcoma, the remaining patient had a thoracic meningioma. Four were male, two female and their mean age was 66 years. CSF was obtained from above and below the block in two patients.

4 Neurological patients CSF was obained from 14 patients with miscellaneous neurological conditions not known to alter CSF neurotransmitters. Nine were male, five female. Eleven of these patients had overnight bedrest and were fasted prior to lumbar puncture. This was performed at 9 a.m. The remaining three patients underwent lumbar myelography. The results from neurological patients have been combined with those from myelogram patients to compare neurotransmitter concentrations between the sexes and at different ages.

5 Spinal drain CSF from nine patients undergoing neurosurgical operation was collected from a spinal drain. Five had aneurysmal subarachnoid haemorrhages one week previously. Two patients had pituitary adenomas, one had a craniopharyngioma and the remaining patient had an orbital tumour. Patients undergoing neurosurgical operations received $10 \mathrm{mg}$ diazepam preoperatively and anaesthesia was induced using thiopentone and maintained with fentanyl, droperidol, alcuronium chloride and nitrous oxide. A spinal drain was inserted at L4/5 after induction of anaesthesia. When the dura was about to be opened, the spinal drain was opened and from 30 to $110 \mathrm{ml}$ of CSF drained. The CSF was collected in $10 \mathrm{ml}$ aliquots.

6 Ventricular CSF Ventricular CSF was obtained during neurosurgical operations from five patients. Three were male, two female. Three patients had hydrocephalus, two secondary to subarachnoid haemorrhage 1 month and many years previously. The other patient had normal pressure hydrocephalus. Another patient had large ventricles from cerebral atrophy resulting from a birth injury and the remaining patient had a frontal lobe glioma. Patients had 10 mg diazepam 1 hour preoperatively and anaesthesia was induced in a similar method to those undergoing other neurosurgical operations.

All the CSF samples were collected onto ice and frozen at $-80^{\circ} \mathrm{C}$ within 10 minutes and were transported in dry ice to LERS-Synthelabo Paris in two batches for analysis. Unfortunately the second batch arrived in Paris in a partially defrosted condition (see below). CSF amino acids were measured with an automated amino acid analyser with fluorescent detection. ${ }^{9}$ The recovery was greater than $85 \%$ as assessed by internal standards. The sensitivity for assays of aspartate, asparagine, glutamate and glutamine were $<0.03$ $\mathrm{nmol}$, and those for taurine, glycine and alanine $<0.1 \mathrm{nmol}$. Variation between assays was $<5 \%$. Results were compared statistically using Student's $t$ test and correlation coefficients.

\section{Results}

Results from the two batches were compared to check for any alterations in amino acid concentrations during transport. Free CSF $\gamma$-aminobutyric acid (GABA) concentrations were greater in batch 2 $(p<0.001)$ whilst aspartate concentrations were lower $(p<0.02)$, there being no differences for other amino acids. These differences may be due to the partial defrosting of specimens that occurred during transportation of batch 2. As it is recognised that free GABA concentrations rise when CSF is left to stand unfrozen because of degradation of homocarnosine, results for aspartate and GABA are therefore reported only for batch 1 .

(a) Comparison of CSF obtained from different sites (fig 1.)

The amino acid concentrations in lumbar myelogram patients are similar to those from the 11 neurological control patients suggesting that fasting, a 9 a.m. puncture, and bedrest are not important factors in determining the amino acid concentrations in CSF.

There were no significant differences in the concentrations of taurine, aspartate, glutamine and GABA between CSF obtained from cervical and lumbar puncture. Asparagine and alanine concentrations were lower in cervical CSF than in lumbar CSF and both amino acids were significantly elevated in CSF from below a spinal block (this suggests that the quantities of alanine and asparagine in the CSF are produced predominantly by the spinal cord). Glycine concentrations showed a fall between lumbar and cervical CSF ( $p<0.025$ ), and there was no significant elevation below a spinal block.

Two patients, one with a mid thoracic spinal block from an extradural secondary from a carcinoma, the second with a $\mathrm{C} 5 / 6$ block from a cervical disc prolapse, had samples of CSF obtained from below and above the block (table 2). Both samples were taken 

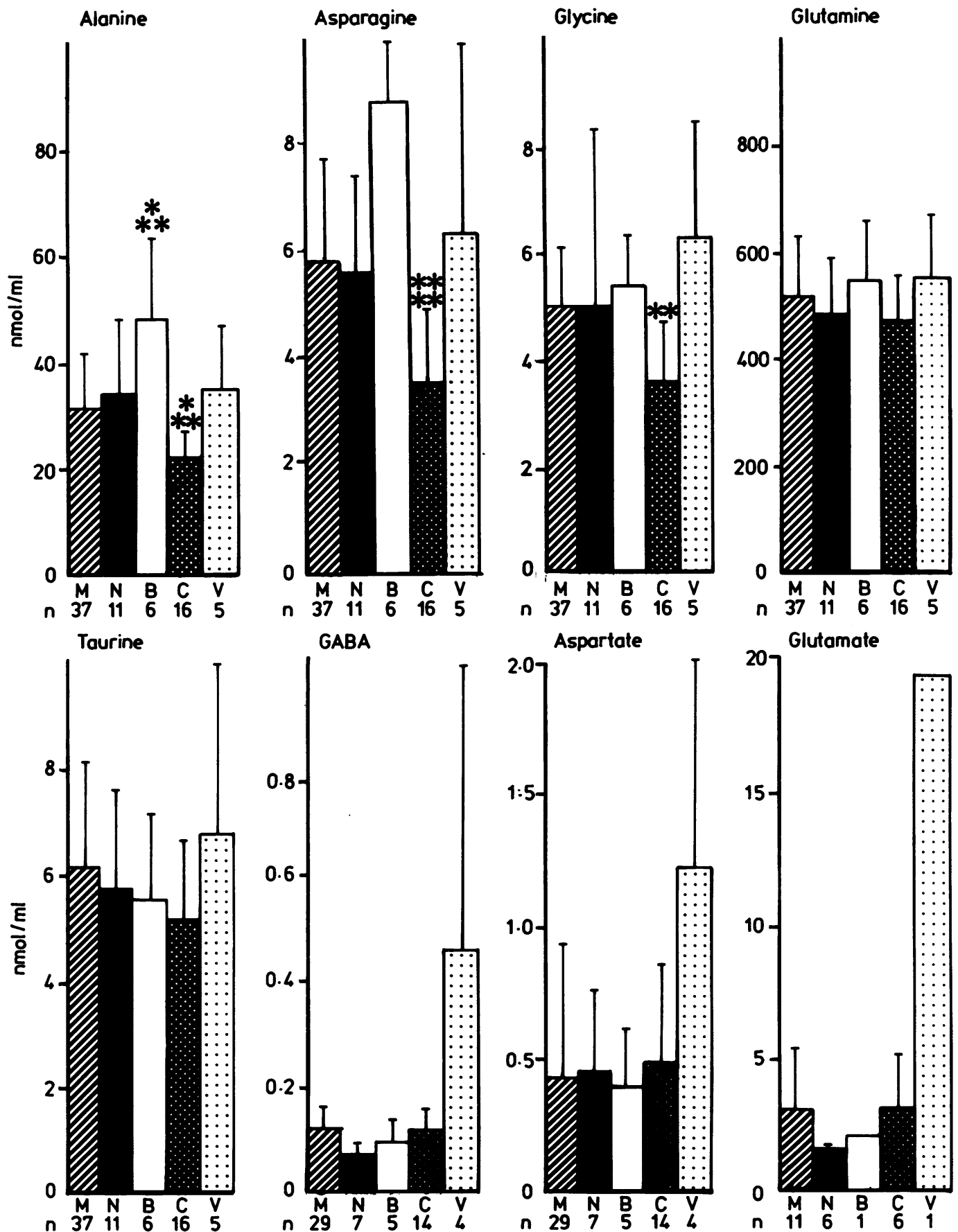

Fig 1 Comparison of CSF amino acid concentrations from different sites, S.D. "M" indicates lumbar CSF from myelogram patients; " $N$ " lumbar CSF from patients with miscellaneous neurological disorders; " $B$ " lumbar CSF from below a complete spinal block; " $C$ " cervical CSF and " $V$ " CSF from a ventricular tap.

$*=p<0.05 ;{ }^{* *}=p<0.025 ;{ }^{* * *}=p<0.005 ;{ }^{* * * *}=p<0.001$

There is no SD bar for glutamate ventricular CSF as only one patient was examined. 

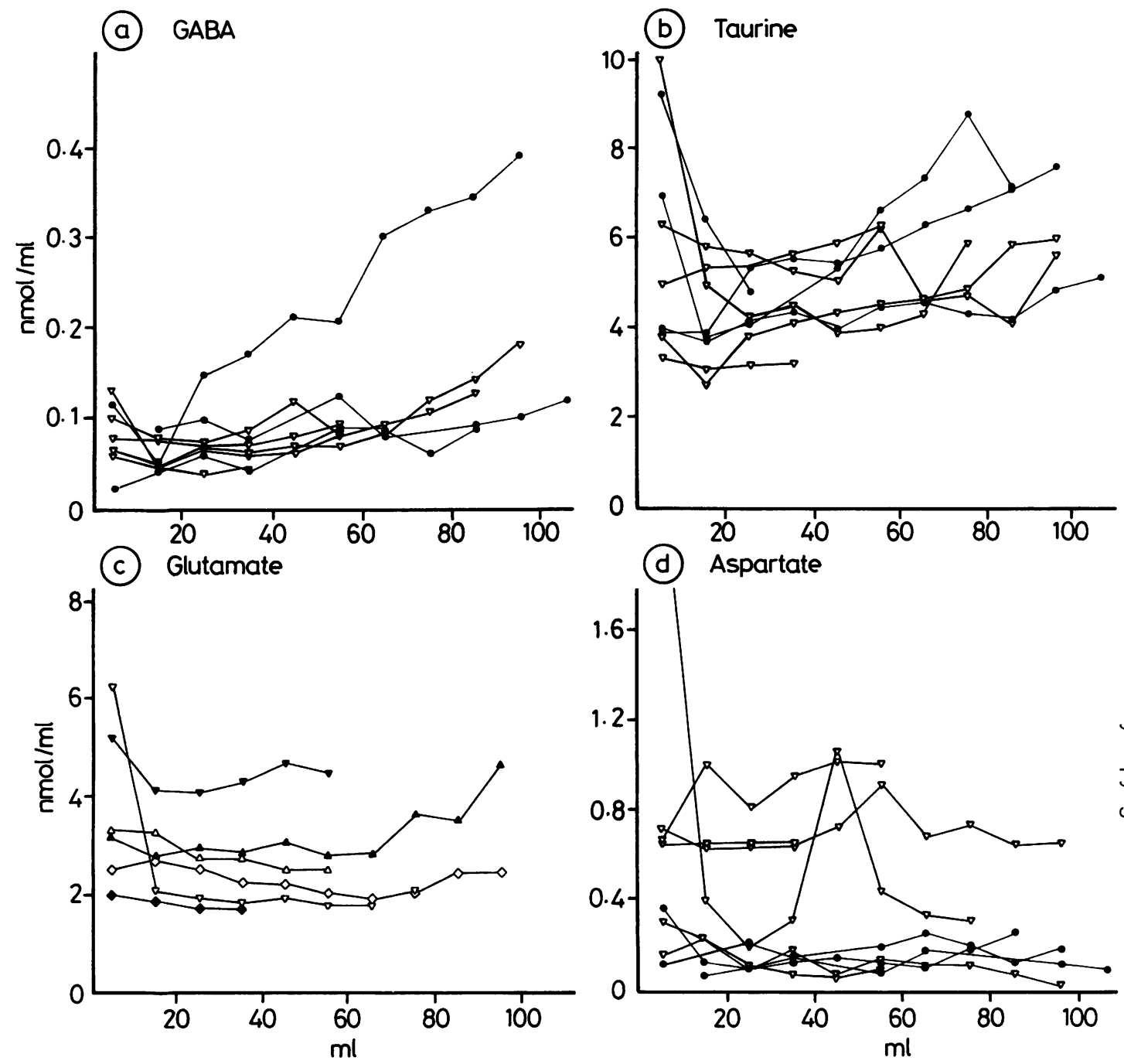

Fig 2 CSF amino acid concentrations from spinal drains in invidivual patients. Open points represent values from patients with subarachnoid haemorrhage, filled points those from non-subarachnoid patients.

$\begin{array}{lcr} & \text { Regression co-efficients } \\ & \text { non-SAH } & \text { SAH } \\ \text { Taurine } & 0.38(\mathrm{p}<0.05) & -0.14 \\ \text { Gaba } & 0.511(\mathrm{p}<0.025) & 0.26 \\ \text { Glutamate } & - & 0.10 \\ \text { Aspartate } & 0.03 & -0.23 \\ \text { Asparagine } & -0.69(\mathrm{p}<0.001) & -0.32 \\ \text { Alanine } & -0.52(\mathrm{p}<0.005) & -0.26 \\ \text { Glycine } & -0.087 & -0.26 \\ \text { Glutamine } & -0.18 & -0.20\end{array}$



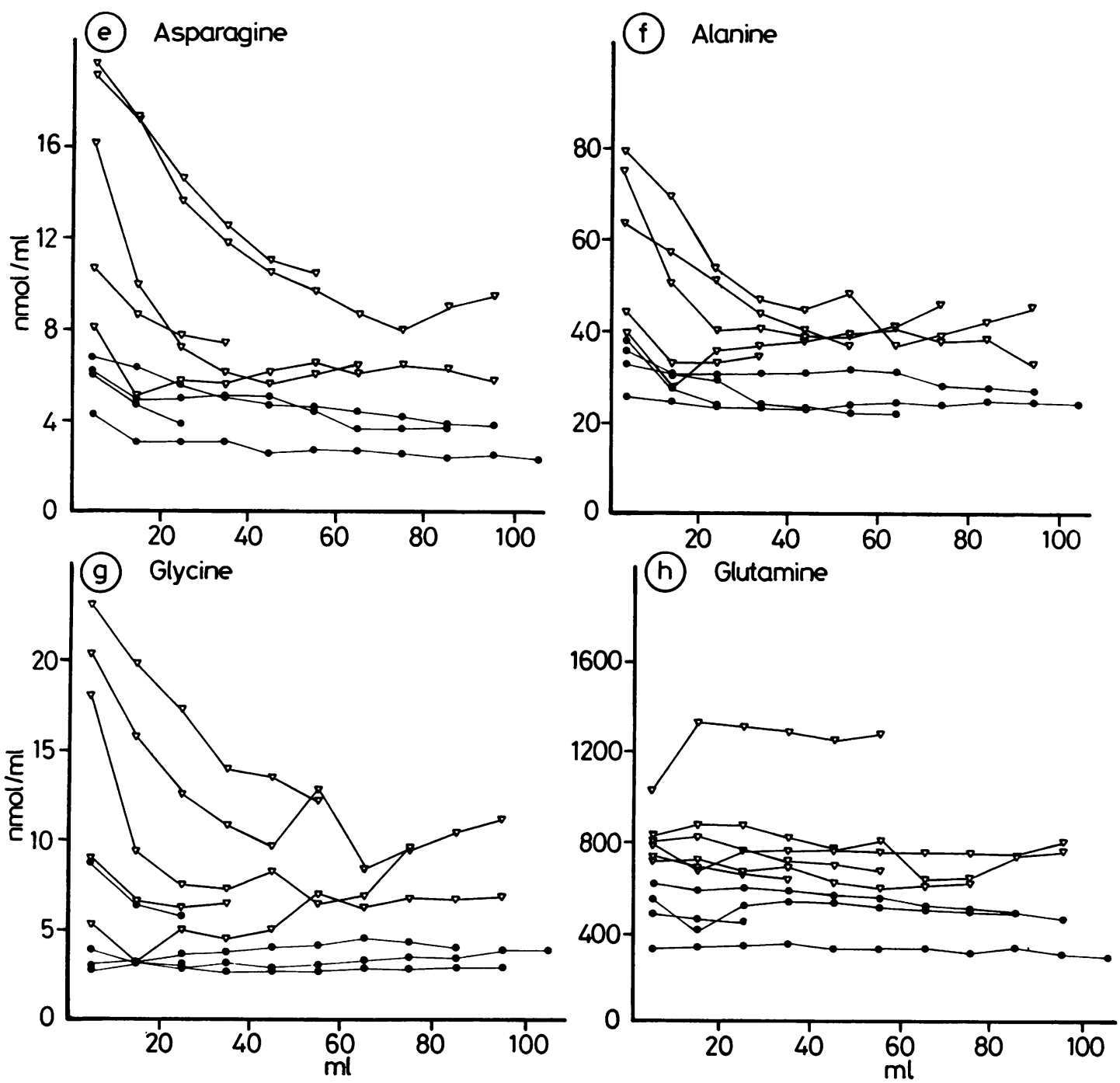

Table 2 CSF above and below a spinal block ( $\mathrm{nmol} / \mathrm{ml})$

\begin{tabular}{lccccccc}
\hline & TAU & ASP & ASN & GLN & GLY & ALA & GABA \\
\hline $\begin{array}{l}\text { Patient 1 } \\
\text { Lumbar }\end{array}$ & 7.72 & 0.416 & 9.07 & 674 & 5.87 & 67.3 & 0.079 \\
$\begin{array}{c}\text { Cervical } \\
\text { Patient 2 }\end{array}$ & 12.80 & 0.292 & 2.40 & 444 & 4.34 & 23.7 & 0.078 \\
$\quad$ Lumbar & & & & & & \\
Cervical & 3.23 & 0.121 & 4.41 & 338 & 4.47 & 27.8 & 0.072 \\
\hline
\end{tabular}

TAU = taurine; $A S P=$ aspartate; $A S N=$ asparagine; GLN = glutamine; GLY = glycine; ALA = alanine 
Table 3 CSF amino acid concentrations ( $\mathrm{nmol} / \mathrm{ml}$, SD) : effects of age and sex

\begin{tabular}{|c|c|c|c|c|}
\hline \multirow[b]{2}{*}{ Amino acid } & \multicolumn{2}{|l|}{ Female } & \multicolumn{2}{|l|}{ Male } \\
\hline & $<40$ & $>40$ & $<40$ & $>40$ \\
\hline $\begin{array}{l}\mathbf{N} \\
\text { Taurine } \\
\text { Asparagine } \\
\text { Glutamine } \\
\text { Glycine } \\
\text { Alanine }\end{array}$ & $\begin{array}{c}6 \\
6 \cdot 07,1 \cdot 49 \\
5 \cdot 25,1 \cdot 10 \\
435,56 \\
3 \cdot 87,1 \cdot 65 \\
28 \cdot 89,6.48\end{array}$ & $\begin{array}{l}17 \\
7.05,2 \cdot 27 \\
5.06,1 \cdot 33^{*} \\
455,81 \dagger \\
5.09,2 \cdot 87 \\
28 \cdot 6,12 \cdot 74^{*}\end{array}$ & $\begin{array}{r}6 \\
4.95,1.09 \\
5.44,1.09 \\
507,87 \\
4.44,2.51 \\
27 \cdot 78,6.48\end{array}$ & $\begin{array}{c}22 \\
5 \cdot 92,1 \cdot 31 \\
6 \cdot 44,2 \cdot 49 \\
559,132 \\
5 \cdot 01,2 \cdot 32 \\
36 \cdot 21,11 \cdot 00\end{array}$ \\
\hline $\begin{array}{l}\text { N } \\
\text { GABA } \\
\text { Aspartate }\end{array}$ & $\begin{array}{l}4 \\
0.133,0.034 \\
0.433,0.471\end{array}$ & $\begin{array}{l}14 \\
0.101,0.038 \\
0.285,0.233\end{array}$ & $\begin{array}{l}4 \\
0.131,0.082 \\
0.686,0.351\end{array}$ & $\begin{array}{l}17 \\
0.094,0.048 \\
0.513,0.590\end{array}$ \\
\hline
\end{tabular}

* $=\mathrm{p}<0.05 ; \dagger=\mathrm{p}<0.005$ for women versus men, both aged over $40 \mathrm{yr}$

within 30 minutes of each other. The results are in keeping with the previously observed trends for asparagine, alanine and glycine.

Aspartate $(p<0.05)$ and GABA concentrations $(p<0.02)$ are greater in ventricular compared with lumbar CSF. There was an increase in aspartate $(p<0.05), \quad$ asparagine $(p<0.025), \quad$ glycine $(\mathrm{p}<0.005)$, alanine $(\mathrm{p}<0.005)$ and GABA $(p<0.025)$ in ventricular compared to cervical CSF.

\section{(b) CSF from spinal drains (fig 2)}

The results of analysis of CSF from spinal drains are summarised in fig 2. CSF concentrations were considerably elevated in specimens from patients with subarachnoid haemorrhage compared with those from patients without subarachnoid haemorrhage for all amino acids except gluatamate, taurine and GABA although similar gradients were observed.

Interestingly the amino acid concentrations in the first $10 \mathrm{ml}$ of CSF obtained were often higher than the subsequent $10 \mathrm{ml}$. Taurine, aspartate, GABA and glycine all had higher values in the first $10 \mathrm{ml}$ compared with the next $10 \mathrm{ml}$. As part of this aliquot will have been present in the spinal drain tubing for up to 1 hour it may be that this particular elevation is artefactual. Therefore the amino acid concentrations for the 10-19th $\mathrm{ml}$ for these amino acids will be used as a baseline for comparisons.

For non-subarachnoid CSF, alanine $(r=0.516$; $\mathrm{p}<0.005)$ and asparagine $(r=0.693 ; \mathrm{p}<0.001)$ show a significant caudorostral gradient. Taurine $(r=0.379 ; \quad p<0.05)$ and GABA $(r=0.511$; $p<0.025)$ concentrations showed a significant rostrocaudal gradient, in the latter case largely due to results from one patient.

\section{(c) Influence of age and sex}

Results from lumbar myelograms and neurological control patients were combined for analysis of the relationship of patients age and sex to CSF amino acid concentrations. Levels of taurine, asparagine, glu- tamine, glycine and alanine all rose with increasing age but only in the case of glycine did this reach statistical significance $(r=0.313 ; p<0.05)$. Free CSF GABA concentrations fell with increasing age $(r=-0.378 ; p<0.05)$. This change was significant in women $(r=-0.645 ; p<0.02)$, but not in men. There was a significant difference in the concentrations of taurine, glutamine and asparagine between the sexes, women having significantly lower concentrations of asparagine $(p<0.05)$ and glutamine $(p<0.001)$, whilst CSF taurine concentrations were significantly elevated in women $(p<0.02)$. If these groups were further subdivided into women or men under or over the age of 40 years, there was no significant difference in the CSF amino acid concentrations between men and women under the age of 40 (table 3). Over the age of 40 , women had significantly lower concentrations of glutamine $(p<0.005)$, alanine $(p<0.05)$ and asparagine $(p<0.05)$ compared to men of the same age.

\section{Discussion}

The control values for lumbar CSF in this study are similar to the majority of studies. There were no significant differences between myelogram and neurological control patients, which indicates that recumbency and overnight fasting do not seem to affect CSF amino acid concentrations in the same way as they affect those of monoamine metabolites. ${ }^{8}$

The age and sex of a patient are of considerable importance, women over the age of $\mathbf{4 0}$ years having significantly lower concentrations of asparagine, glutamine and alanine as compared with men of a similar age. There was also a significant correlation between glycine (concentration increasing with age) and GABA (concentration decreasing with age) and age. McGale et al with 37 control patients, found a significant correlation between age and glutamine $(p<0.05)$ and glycine concentrations $(p<0.01)$ but were unable to detect any difference between the 
sexes. ${ }^{1}$ Engelson and Elsayad with 30 patients did not detect a significant difference in amino acid concentrations between the sexes or patients over and under the age of $35 .^{2}$ In this study there was a significant fall in free CSF GABA concentrations with increasing age only in women. Hare et al reported that lumbar CSF GABA fell with increasing age and that there was a significant difference in free CSF GABA between women and men. ${ }^{10}$ This difference increased with increasing age. They also found a significant difference in free CSF GABA between women under and over the age of $40 .{ }^{10}$

Previous studies have demonstrated a rostrocaudal gradient for GABA. ${ }^{11} 12$ Wood et al obtained $40 \mathrm{ml}$ of CSF at lumbar puncture and analysed sequential $1 \mathrm{ml}$ aliquots, demonstrating a significant rostrocaudal gradient for free GABA. ${ }^{11}$ Grove et al (five patients) compared free and total CSF GABA concentrations in $30 \mathrm{ml}$ of CSF from lumbar puncture. ${ }^{12}$ A significant rostrocaudal gradient occurred for both free and total GABA. Analysis of simultaneous suboccipital and lumbar CSF samples in four more patients failed to demonstrate a significant difference between the samples. This study also confirmed the presence of a rostrocaudal gradient for GABA in specimens from spinal drains.

A decreasing concentration gradient between the brain and lumbar CSF compartments suggests a major contribution from brain GABA to lumbar CSF GABA content. ${ }^{12}$ CSF taurine concentrations from spinal drains also show a similar rostrocaudal gradient. Aspartate concentrations from spinal drains and cervical CSF showed a trend towards higher concentrations rostrally though this failed to reach statistical significance.

Alanine and asparagine show the reverse, that is, a caudorostral gradient. Levels of these two amino acids fell significantly in CSF obtained from spinal drains. This reverse gradient also occurred in myelogram patients, there being a significant difference in concentrations between lumbar and cervical samples. Furthermore, the concentrations of these two amino acids were higher in lumbar CSF obtained from below a spinal block as compared with that from above the block. This suggests that the CSF concentrations of these two amino acids originate predominantly from the spinal cord.

The results from the ventricular taps are difficult to interpret as patients may have disturbed CSF dynamics. Ventricular GABA concentrations were significantly elevated compared with cervical and lumbar samples. Ventricular taurine concentrations were higher than in lumbar and cervical samples and significantly elevated if compared with the 10-19th $\mathrm{ml}$ from spinal drains. Ventricular aspartate concentrations were greatly raised as compared to CSF obtained from other sites. Asparagine, alanine and glycine concentrations were also significantly elevated in ventricular CSF compared with cervical samples. In CSF from ventricular taps in 14 patients McGale et al found a significant elevation in glycine $(p<0.01)$ and a reduction of glutamine concentrations $(p<0.01)$ compared to lumbar samples (37 patients). ${ }^{1}$ Both the anaesthesia and surgery during ventricular taps may produce altered CSF dynamics.

Inevitably in such data some conflicts exist. For glycine lower concentrations were found in cervical than lumbar CSF, but no gradient was apparent in spinal drain data. Conversely for GABA and taurine a rostrocaudal gradient was apparent in data from spinal drains, but not in comparison with lumbar and cervical puncture CSF (though a trend in this direction was apparent for taurine).

In conclusion, there are differences in amino acid concentrations with the age and sex of patients. Such factors should be carefully controlled in future clinical studies. There is a rostrocaudal gradient for GABA and taurine, suggesting they originate centrally, and caudorostral for alanine and asparagine suggesting a spinal origin in the CSF. Lumbar CSF GABA and taurine concentrations appear to reflect cerebral metabolism to a greater degree than other amino acids. Any alterations in these latter amino acid concentrations cannot easily be extrapolated to cerebral disturbances.

We thank Dr N R Clitheroe and Dr E K S Smith and the Neuroradiology department for collection of samples and the Neuropathology department for their help in storage and transportation of samples.

\section{References}

1 McGale EHF, Pye IF, Stonier C, Hutchinson EC, Aber GM. Studies of the inter-relationship between cerebrospinal fluid and plasma amino acid concentrations in normal individuals. J Neurochem 1977;29:291-7.

2 Engelson B, Elsayed S. Increased concentrations of aspartic acid in the cerebrospinal fluid of patients with epilepsy and trigeminal neuralgia: an effect of medications. Acta Neurol Scand 1984;70:70-6.

3 Mutani R, Monaco F, Durelli L, Delsedime M. Free amino acids in the cerebrospinal fluid of epileptic subjects. Epilepsia 1974;15:593-7.

4 Plum CM. Free amino acid levels in the cerebrospinal fluid of normal humans and their variation in cases of epilepsy and Spielmeyer-Vogt-Batten Disease. $J$ Neurochem 1974;23:595-600.

5 Gjessing LR, Gjesdal P, Sjaastad $O$. The free amino acids in human cerebrospinal fluid. $J$ Neurochem 1972;19:1807-8.

6 Perry TL, Hansen S, Diamond S, Stedman D. Plasma amino acid levels in Huntington's chorea. Lancet 1969;1:806-8. 
7 Van Sande M, Mardens Y, Adriaenssens K, Lowental A. The free amino acids in human cerebrospinal fluid. $J$ Neurochem 1970;17:125-35.

8 Garelis E, Sourkes TL. Use of cerebrospinal fluid drawn at pneumoencephalography in the study of monoamine metabolism in man. J Neurol Neurosurg Psychiatry 1974;37:704-10.

9 Bohlen P, Schoeder R. High sensitivity amino acid analysis: methodology for the determination of amino acid compositions under less than 100 picomoles of peptides. Anal Biochem 1982;126:144-50.

10 Hare TA, Wood JH, Manyam NVB et al. Central nervous system-aminobutyric acid activity in man related to age and sex as reflected in cerebrospinal fluid Arch Neurol 1982;39:247-9.

11 Wood JH, Hare TA, Enna SJ, Manyam NVB. Sites of origin and rostrocaudal concentration gradients of GABA in cerebrospinal fluid. Brain Res Bull 1980;5:Suppl.2:111-4.

12 Grove J, Schechter PJ, Hanke NFJ et al. Concentration gradients of free and total-aminobutyric acid and homocarnosine in human CSF: Comparison of suboccipital and lumbar sampling. $J$ Neurochem 1982; 39:1618-22. 